\title{
Influência da dieta sobre a abundância de Moenkhausia dichroura (Characiformes, Characidae) no reservatório de Manso, Estado de Mato Grosso
}

\author{
Marlene R. da Silva ${ }^{1} \&$ Norma S. Hahn ${ }^{1,2}$
}

1. Programa de Pós-Graduação em Ecologia de Ambientes Aquáticos Continentais, Universidade Estadual de Maringá, Maringá, PR, Brasil. (marleners@ibest.com.br)

2. Núcleo de Pesquisas em Limnologia, Ictiologia e Aquicultura, Universidade Estadual de Maringá, Av. Colombo 5790, 87020-900 Maringá, PR, Brasil. (hahnns@ nupelia.uem.br)

\begin{abstract}
Diet influencing abundance of Moenkhausia dichroura (Characiformes, Characidae) in Manso Reservoir, State of Mato Grosso, Brazil. This study aimed to analyze the diet and abundance of Moenkhausia dichroura Kner, 1858, after the filling of Manso Reservoir, State of Mato Grosso, Brazil. The diet was compared spatially (upstream, reservoir and downstream) and temporally (phase I, first year after damming and phase II, fourth year after damming). Stomach contents of 392 fishes were analyzed and the diet was described through volumetric representation of the food categories. Upstream the reservoir this species showed tendency to insectivory ( $\mathrm{V} \%=51.46$ of aquatic insects in phase I and $\mathrm{V} \%=56.07$ of terrestrial insects in phase II). In the Reservoir it showed tendency to zooplanktivory ( $\mathrm{V} \%=77.11$ and $\mathrm{V} \%=64.73$ of microcrustaceans, in phases I and II, respectively) whereas downstream the reservoir to herbivory ( $\mathrm{V} \%=56.02$ and $\mathrm{V} \%=62.84$ of plants in phases I and II, respectively). We verified significant spatial differences in the diet (Kruskal-Wallis; $H=197.11, p<0.05$ ). Significant temporal differences was verify between diet of the individuals of upstream I and upstream II (test a posteriori - multiple comparison; $p<0.05$ ). There was an abrupt increase of $M$. dichroura abundance during the studied period, mainly during the fourth year after damming, inside the reservoir. This fact seems closely related to the high abundance and availability of zooplankton in the dammed environment in addition to the ability of the species to exploit this resource.
\end{abstract}

KEYWORDS. Diet, fish, trophic opportunism, dam environmental.

RESUMO. Este estudo teve por objetivo avaliar a dieta e abundância de Moenkhausia dichroura Kner, 1858 após a formação do reservatório de Manso, Estado de Mato Grosso. A dieta foi comparada espacial (montante, reservatório e jusante) e temporalmente (fase I, primeiro ano do represamento e fase II, quarto ano do represamento). Conteúdos estomacais de 392 indivíduos foram analisados e a dieta foi descrita através da representação volumétrica dos recursos alimentares. A montante a espécie mostrou tendência à insetivoria $(\mathrm{V} \%=51,46$ de insetos aquáticos na fase $\mathrm{I}$ e $\mathrm{V} \%=56,07$ de insetos terrestres na fase II), no reservatório à zooplanctivoria $(\mathrm{V} \%=77,11$ e V\% $=64,73$ de microcrustáceos, nas fases I e II, respectivamente) e a jusante à herbivoria (V\% $=56,02$ e V\% $=62,84$ de vegetais, nas fases I e II, respectivamente). Constatou-se diferença espacial significativa na dieta (Kruskal-Wallis; $H=197,11, p<0,05$ ), sendo também observada diferença temporal significativa entre as dietas dos indivíduos das estações montante I e montante II (teste a posteriori de comparação múltipla; $p<0,05$ ). Houve um aumento abrupto na abundância de $M$. dichroura durante o período de estudos, principalmente no quarto ano do represamento, no corpo principal do reservatório. Este fato parece estar estreitamente relacionado com a alta abundância e disponibilidade do zooplâncton no ambiente represado e também com a habilidade da espécie em explorar este recurso.

PALAVRAS-CHAVE. Dieta, peixe, oportunismo trófico, ambiente represado.

A construção de reservatórios tem aumentado de forma expressiva nos últimos anos e estes são hoje componentes indissociáveis da paisagem brasileira (BenEdito-Céílio et al., 1997; AgostinHo et al., 2007) e, independente de sua finalidade, modificam os ecossistemas naturais. A transformação do ambiente lótico em lêntico é uma das consequências ecológicas mais importantes dos represamentos, pois altera as condições físicas, químicas e biológicas do ambiente, refletindo de forma impactante sobre toda a biota local (JúLIO JR. et al., 1997).

$\mathrm{O}$ entendimento preciso das alterações impostas pela formação de um reservatório, durante seu enchimento e no período subsequente é fundamental para definição de ações de manejo (Agostinho et al., 2007), visto que, de acordo com WoYNAROvich (1991), a alteração da composição ictiofaunística é a que reflete maior importância econômica.

Logo após o barramento do fluxo natural da água, os organismos presentes sofrem repentino impacto ambiental, devido à fase inicial de enchimento (AgostinHo et al., 2007). Isso acarreta uma crítica e imprevisível fase de colonização e reestruturação das comunidades presentes (AgostinHo et al., 1999; HAHN \& FUGI, 2007), as quais precisam se adequar à complexidade dos distúrbios.

Uma das consequências inevitáveis dos represamentos sobre a fauna aquática é a alteração na composição e abundância relativa das espécies de peixes (AgostinHo et al., 1999), a qual é afetada pela proliferação de espécies oportunistas, compostas por indivíduos de pequeno porte e de baixo valor comercial, em detrimento daquelas migradoras de grande porte (AgostinHo et al., 1992; HAHN \& FuGI, 2007). Outro fator que merece destaque é o oportunismo trófico de certos peixes, que tiram proveito de uma fonte alimentar vantajosa em determinado momento. Esse é um caso comum entre espécies filtradoras que tem a capacidade (rastros branquiais modificados) de aproveitar o incremento do zooplâncton no início de formação dos reservatórios (FERREIRA, 1984; SANTOS, 1995; Benedito-Cecílio \& Agostinho, 1999; CAssemiro et al., 2003; HAHN \& Fugi, 2007).

No reservatório de Manso, dentre as espécies capturadas, Moenkhausia dichroura Kner, 1858, foi a 
única a apresentar rastros branquiais modificados, os quais foram descritos por BenINE et al. (2004) como adaptados à filtração. Estudos referentes à dieta indicam tratar-se de uma espécie que se alimenta de insetos e zooplâncton dependendo do ambiente (Poullly et al., 2003; REJAS et al., 2005).

Neste estudo foi investigada a dieta de $M$. dichroura e sua abundância após a construção do reservatório de Manso, Estado de Mato Grosso, Brasil. Dessa forma, procurou-se comparar temporal e espacialmente os tipos de alimento consumidos por indivíduos coletados no primeiro e quarto anos do represamento, no corpo principal do reservatório, à montante e à jusante, discutindo-se também se a dieta influenciou a abundância da espécie.

\section{MATERIAL E MÉTODOS}

Área de estudo. O rio Manso, principal formador do rio Cuiabá $\left(14^{\circ} 32^{\prime}-15^{\circ} 40^{\prime} \mathrm{S}\right.$ e $\left.54^{\circ} 40^{\prime}-55^{\circ} 55^{\prime} \mathrm{W}\right)$, foi barrado em 1999 para formação do reservatório de Manso, Estado de Mato Grosso, Brasil, localizado próximo ao Parque Nacional da Chapada dos Guimarães. O reservatório inundou, além da montante da barragem, as porções inferiores dos rios Casca, Palmeiras e Quilombo, totalizando uma área de $427 \mathrm{~km}^{2}$ na cota máxima. Cerca de $80 \mathrm{~km}$ após a confluência com o rio Casca, o rio Manso se junta ao rio Cuiabazinho, formando o rio Cuiabá, que segue a partir daí por regiões mais baixas, com densa rede de drenagem e regime regular de chuvas desaguando no Pantanal de Mato Grosso, que se estende além da confluência dos rios Cuiabá com o Paraguai (dados não publicados).

Os peixes utilizados neste estudo foram coletados em três estações com diversos pontos de amostragens: dois à montante do reservatório; seis no corpo principal e três à jusante do reservatório (Fig. 1).

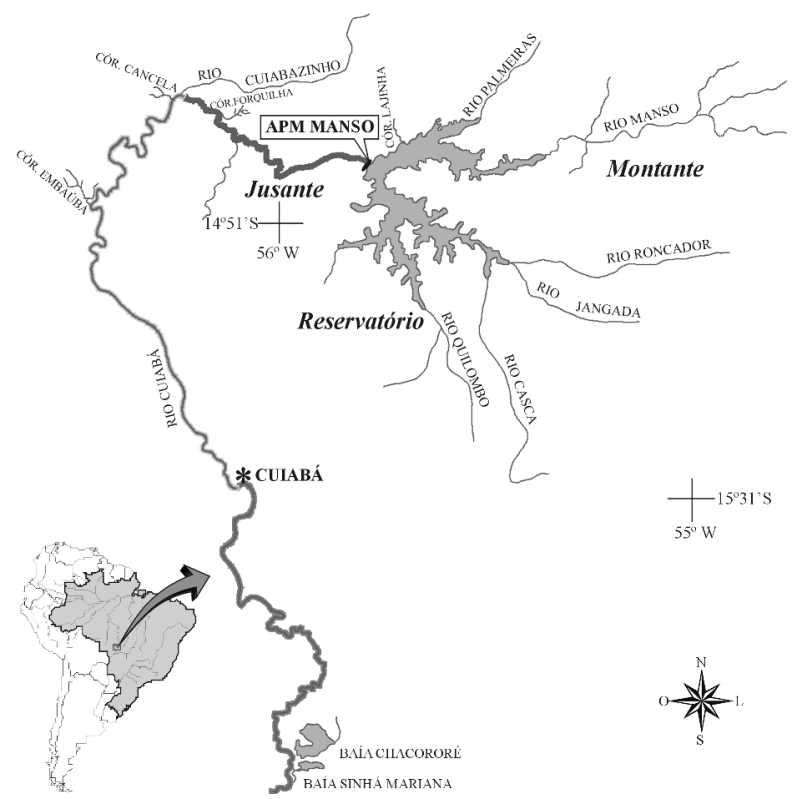

Figura 1. Área de estudo, destacando as estações de coleta no corpo principal do reservatório de Manso, Estado de Mato Grosso, à montante e à jusante.
Amostragens. Os peixes foram amostrados mensalmente no período de março de 2000 a fevereiro de 2001 (fase I) e entre março de 2003 a fevereiro de 2004 (fase II), utilizando-se baterias de redes de espera, com diferentes malhas, variando entre 2,4 a $10 \mathrm{~cm}$ entre nós opostos, que ficaram expostas por 24 horas, com revistas ao amanhecer $(8 \mathrm{~h})$, ao entardecer $(16 \mathrm{~h})$ e à noite $(22 \mathrm{~h})$. Adicionalmente foram utilizadas redes de arrasto, a montante e no corpo do reservatório, com malha de um $\mathrm{cm}$ entre nós opostos. Após as amostragens, os peixes foram identificados, pesados, medidos e os estômagos fixados em formol 4\%. Exemplares testemunhos foram depositados na Coleção de Peixes do Núcleo de Pesquisas em Limnologia, Ictiologia e Aquicultura (Nupélia) da Universidade Estadual de Maringá, PR, Brasil (NUP 3128).

Análise dos dados. Dieta. Os conteúdos estomacais foram analisados sob microscópio estereoscópico e óptico. Para avaliação quantitativa dos recursos alimentares, utilizou-se o método volumétrico, onde o volume de cada item alimentar é registrado, obtendo-se a porcentagem em relação ao volume total de todos os conteúdos estomacais (Hyslop, 1980). O volume foi obtido através de placa milimetrada e posteriormente transformado em $\mathrm{ml}$ (Hellawel \& Abel, 1971).

Análise estatística. Para avaliar mudanças na dieta, uma combinação de fatores espaciais (montante, reservatório e jusante) e temporais (fase I, primeiro ano após o enchimento; fase II, quarto ano após o enchimento) foram ordenados de acordo com uma análise de correspondência destendenciada (DCA - Detrended Correspondence Analysis; Hill \& GAUCH JR., 1980). Para essa ordenação foram usados os dados de volume de todos os conteúdos estomacais, estimados individualmente, os quais foram transformados em raiz quadrada devido à heterogeneidade dos dados. A DCA é uma análise de correspondência, na qual os eixos formam uma combinação linear de variáveis explanatórias (PoulLly et al., 2003). A fim de verificar possíveis diferenças espaciais e temporais significativas na dieta, foi utilizado o teste não-paramétrico de Kruskal-Wallis, uma vez que os pressupostos da análise de variância (one-way) não foram atendidos. $\mathrm{O}$ teste a posteriori de comparação múltipla foi aplicado com o objetivo de verificar onde ocorreram as diferenças.

As análises estatísticas e gráficas foram feitas usando o software Statistica ${ }^{\mathrm{TM}}$ for Windows exceto a DCA, a qual foi realizada no programa PC-ORD 4.0 for Windows (McCune \& Mefford, 1999). O nível de significância estatística adotado foi $p<0,05$.

Captura. Para a estimativa espacial e temporal de captura da espécie, foram utilizados apenas os indivíduos capturados com redes de espera e a estes foram aplicados cálculos de captura por unidade de esforço, CPUE $=\left({ }^{\mathrm{n} i} / \mathrm{E}\right) * 1000 \mathrm{~m}^{2}$; onde, n é o número de indivíduos removidos na amostragem do tempo $i$ e Eé o tamanho do esforço de captura (metros ${ }^{2}$ de rede/hora de exposição) empreendido na amostragem do tempo $i$ x 1000. 


\section{RESULTADOS}

Um total de 392 estômagos (82 de indivíduos provenientes da montante, 242 do reservatório e 68 da jusante) foi analisado, contemplando todos os meses de coleta.

$\mathrm{Na}$ montante os peixes se comportaram como insetívoros, consumindo predominantemente insetos aquáticos $(\mathrm{V} \%=51,46 \%)$ na fase I e insetos terrestres $(\mathrm{V} \%=56,07 \%)$ na fase II; no reservatório os peixes forragearam quase que exclusivamente sobre o zooplâncton, sendo esta categoria representada por uma proporção volumétrica de $77,11 \%$ na fase I e de $64,73 \%$ na fase II. Na estação jusante, os peixes mostraram tendência à herbivoria, sendo que vegetal terrestre somou $56,02 \%$ do volume da dieta na fase I e $62,84 \%$ na fase II (Tab. I).

Os resultados da ordenação da dieta, através da DCA (Fig. 2) foram interpretados particularmente pelo eixo 1, o qual explicou a maior parte da variabilidade dos dados (autovalor $=0,68$ ). A distribuição dos pontos ao longo do eixo 1 mostra que a dieta foi distinta entre as três estações de coleta, sendo que vegetal terrestre e zooplâncton foram os recursos alimentares que mais influenciaram a distribuição dos dados, nos escores à direita e à esquerda, respectivamente. As médias obtidas através dos escores do eixo 1 da DCA (Fig. 3) confirmam que a dieta da espécie foi espacialmente diferente, independente das fases consideradas, com exceção da montante, onde notase um distanciamento entre as médias, devido ao consumo diferenciado de insetos aquáticos na fase I e de insetos terrestres na fase II. Constatou-se diferença espacial significativa na dieta $(H=197,11 ; p<0,05)$, sendo que o teste a posteriori indicou também diferença temporal significativa entre as dietas dos indivíduos das estações montante I e montante II $(p<$ $0,05)$.

Os dados de captura apontaram para um incremento temporal nas três estações de coleta, porém, no corpo principal do reservatório, onde a captura da espécie representava cerca de 300 indivíduos $/ 1000 \mathrm{~m}^{2}$ de rede no primeiro ano, passou para mais de 3500 indivíduos/1000 $\mathrm{m}^{2}$ de rede no quarto ano do represamento, evidenciando uma explosão populacional (Fig. 4).

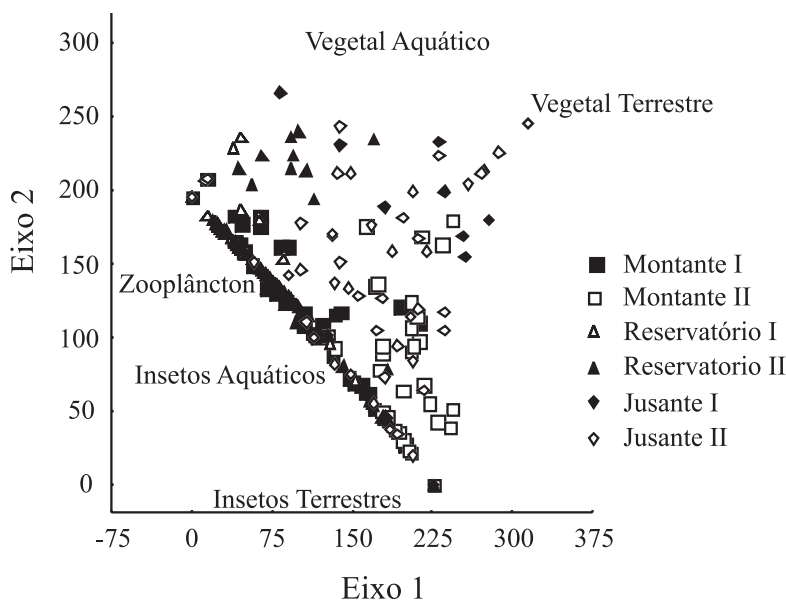

Figura 2. Análise de Correspondência Destendenciada (DCA), aplicada sobre os dados da dieta de Moenkhausia dichroura Kner, 1858, considerando as estações de coleta (montante, reservatório e jusante) e fases de formação do reservatório de Manso, Estado de Mato Grosso, Brasil (fase I, primeiro ano do represamento; fase II, quarto ano do represamento).

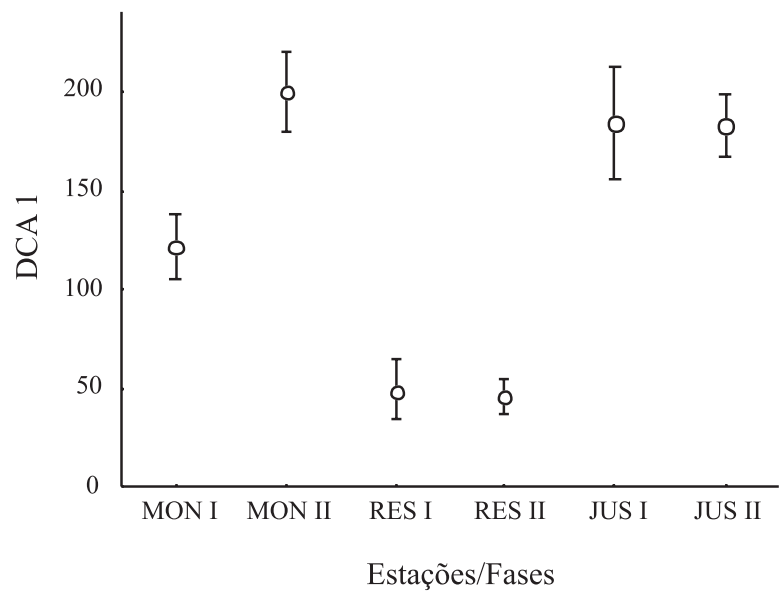

Figura 3. Valores médios do eixo 1 da DCA (Análise de Correspondência Destendenciada), aplicada aos dados de volume dos recursos alimentares consumidos por Moenkhausia dichroura Kner, 1858, nas diferentes estações de coleta e fases de formação do reservatório de Manso, Estado de Mato Grosso, Brasil (I, primeiro ano do represamento; II, quarto ano do represamento; JUS, jusante; MON, montante; RES, reservatório)

Tabela I. Valores de volume ( $\mathrm{ml}$ ), representando a importância dos recursos alimentares na dieta de Moenkhausia dichroura Kner, 1858, nas diferentes estações de coleta e fases de formação do reservatório de Manso, Estado de Mato Grosso, Brasil (I, primeiro ano do represamento; II, quarto ano do represamento. Números em negrito representam o alimento principal).

\begin{tabular}{lcccccr}
\hline & Montante I & MontanteII & Reservatório I & Reservatório II & JusanteI & Jusante II \\
\hline Zooplâncton & 16,87 & & $\mathbf{7 7 , 1 1}$ & $\mathbf{6 4 , 7 3}$ & 1,88 & 7,55 \\
Insetos aquáticos & $\mathbf{5 1 , 4 6}$ & 37,50 & 13,24 & 19,50 & 17,67 & 14,95 \\
Insetos terrestres & 29,95 & $\mathbf{5 6 , 0 7}$ & 8,35 & 14,00 & 19,55 & 11,18 \\
Vegetal aquático & 0,34 & 1,96 & 1,20 & 1,43 & 4,89 & 3,47 \\
Vegetal terrestre & 1,38 & 4,46 & 0,07 & 0,33 & $\mathbf{5 6 , 0 2}$ & $\mathbf{6 2 , 8 4}$ \\
\hline
\end{tabular}




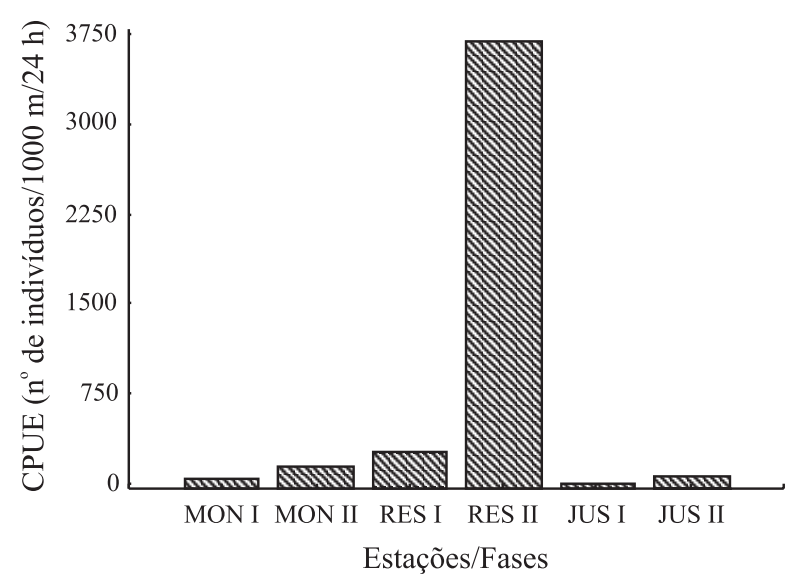

Figura 4. Abundância numérica (CPUE) de Moenkhausia dichroura Kner, 1858, nas diferentes estações de coleta e fases de formação do reservatório de Manso, Estado de Mato Grosso, Brasil (I, primeiro ano do represamento; II, quarto ano do represamento; JUS, jusante; MON, montante; RES, reservatório).

\section{DISCUSSÃO}

Neste estudo, a composição da dieta de $M$. dichroura diferiu entre as estações de coleta, contudo, foi similar temporalmente (com exceção da Montante), quando considerados o primeiro e quarto ano após o represamento. Embora na Montante a espécie tenha apresentado hábito insetívoro, houve mudança temporal significativa na origem do recurso, de autóctone (insetos aquáticos) para alóctone (insetos terrestres), indicando sua capacidade em explorar diferentes compartimentos da coluna d'água.

O consumo de invertebrados (principalmente insetos aquáticos e terrestres) por $M$. dichroura foi também descrito por PouILLY et al. (2004) na planície de inundação do rio Mamoré. Adicionalmente, SAZIMA (1986), Peretti \& Andrian (2004) e Poullly et al. (2006) mencionam os mesmos tipos de recursos na dieta de Moenkhausia intermedia em ambientes aquáticos não impactados, levando a crer que este seja o regime alimentar mais comum para espécies deste gênero.

No reservatório, $M$. dichroura maximizou sua dieta quase que exclusivamente sobre o zooplâncton. Ao contrário de muitos organismos, para os quais a formação de reservatórios é prejudicial, para este grupo de invertebrados, este evento é uma oportunidade para colonização, visto que podem se desenvolver em curtos períodos de tempo após o represamento (Rocha et al., 1999). Segundo BAXTER (1977), uma das alterações esperadas em reservatórios é o aumento das comunidades zooplanctônicas, como consequência do incremento na produtividade primária. Assim, é esperado que muitas populações de peixes se beneficiem desse recurso, embora, somente algumas tiram vantagem dessa oportunidade em reservatórios recém-represados (FERREIRA, 1984; LANSAC-TôHa et al., 1991; SANTOS, 1995; Abujanra \& Agostinho, 2002; Cassemiro et al., 2003; HaHn \& Fugi, 2007). Esse fato se deve, em grande parte, ao modo de forrageamento de muitas espécies e à capacidade de reter o zooplâncton nos rastros branquiais, os quais devem ser adaptados à filtração. MоттA et al. (1995), associam positivamente o comprimento dos rastros e a quantidade de zooplâncton consumido. Dessa forma, com as prerrogativas de abundância do alimento (zooplâncton) e dos requisitos morfológicos favoráveis (rastros branquiais longos), $M$. dichroura foi a única espécie dentre as 127 previamente estudadas no reservatório de Manso (dados não publicados) que conseguiu captar plenamente esse tipo de alimento no novo ambiente.

Nas três estações estudadas, a abundância de $M$. dichroura se alterou temporalmente, embora tenha sido no reservatório o ambiente em que a espécie sofreu incremento mais expressivo. BenINE (2004) comenta que esta espécie pode dobrar seu tamanho populacional em menos de quinze meses, tempo que proporciona vantagem ecológica à espécie, denominada por PAULY (1980) como $r$-estrategista nos atributos reprodutivos. Assim, acreditase que a espécie tenha acumulado energia em biomassa para investir nos atributos reprodutivos o que consequentemente desencadeou seu incremento populacional. Este fato está de acordo com a teoria de forrageamento ótimo, a qual faz referência ao ganho máximo de energia líquida, através da alimentação, com o objetivo de maximizar o sucesso reprodutivo (GERKING, 1994). A esse respeito, Poullzy et al. (2003) afirmam que a dieta dos peixes depende não somente da disponibilidade do alimento como também da capacidade do peixe para capturá-lo e utilizá-lo. No Reservatório, $M$. dichroura comportou-se como forrageadora de zooplâncton, tirando proveito de uma fonte alimentar abundante e acessível. A despeito de seus rastros branquiais serem longos, unidos e com pequenos espinhos (BENINE et al., 2004), ela utiliza outros tipos de alimento dependendo do local onde se encontra (Pouilly et al., 2003; Rejas et al., 2005), tratando-se, portanto, de uma espécie zooplanctívora facultativa.

Rocha et al. (1995) enfatizaram que modificações na estrutura e na dinâmica da comunidade zooplanctônica podem produzir mudanças em toda a estrutura trófica de um reservatório. Dessa forma, espécies que conseguem aproveitar bem os recursos de origem interna do reservatório (zooplâncton, insetos, outros invertebrados detritos e peixes) prevalecem em número e biomassa (Agostinho \& Zalewski, 1995; Araúso-Lima et al., 1995; Agostinho et al., 1999).

Infere-se, portanto, que o impacto promovido pela formação de reservatórios, desencadeia mudanças estruturais nas cadeias alimentares, com reflexos em seus níveis inferiores ("top down") e superiores ("bottom up"). Neste sentido, é previsto que $M$. dichroura tenha interferido nos mecanismos "top down" que estruturavam a cadeia trófica antes da formação do reservatório, visto que também influenciou significativamente a dieta de Acestrorhynchus pantaneiro Menezes, 1992 (dados não publicados), uma espécie piscívora que se alimentou quase que exclusivamente de $M$. dichroura, após sua explosão populacional no reservatório de Manso. 
Agradecimentos. Os autores agradecem ao Nupélia e ao Programa de Pós-Graduação em Ecologia de Ambientes Aquáticos Continentais da Universidade Estadual de Maringá, pelo apoio financeiro e logístico durante a realização deste trabalho. À CAPES pela concessão de bolsa ao primeiro autor.

\section{REFERÊNCIAS BIBLIOGRÁFICAS}

Abujanra, F. \& Agostinho, A. A. 2002. Dieta de Hypophthalmus edentatus (Spix, 1829) (Osteichthyes, Hypophthalmidae) e variações de seu estoque no reservatório de Itaipu. Acta Scientiarum 24(2):401-410.

Agostinho, A. A.; Gomes, L. C. \& Pelicice, F. M. 2007. Ecologia e manejo de recursos pesqueiros em reservatórios do Brasil. Maringá, EDUEM. 501p.

Agostinho, A. A.; Júlio JR., H. F. \& Borghetti, J. R. 1992 Considerações sobre os impactos dos represamentos na ictiofauna e medidas para sua atenuação. Um estudo de caso: reservatório de Itaipu. Revista Unimar 14:89-107.

Agostinho, A. A.; Miranda, L. E.; Bini, L. M.; Gomes, L. C.; Thomaz, S. M. \& Suzuki, H. I. 1999. Patterns of colonization in neotropical reservoirs, and prognoses on aging. In: TUNDISI, J. G. \& Straskraba, M. eds. Theoretical reservoir ecology and its applications. São Carlos, International Institute of Ecology; Leiden, The Netherlands, Brackhuys Publishers; Rio de Janeiro, Brazilian Academy of Sciences. p.227-265.

Agostinho, A. A. \& Zalewsky, M. 1995. The dependence of fish community structure and dynamics on floodplain and riparian ecotone zone in Paraná River Brazil. Hydrobiologia 303(1$3): 141-148$

Araújo-Lima, C. A. R. M.; Agostinho, A. A. \& Fabré, N. N. 1995 Trophic aspects of fish communities in brazilian rives and reservoirs. In: Tundisi, J. B.; Bicudo, C. E. M. \& MatsumuraTundisı, T. eds. Limnology in Brazil. São Paulo, ABC/SBL. p.105-136.

BAXTER, R. M. 1977. Environmental effects of dams and impoundments. Annual Review of Ecology and Systematics 8:255-283.

Benedito-Cecílio, E. \& Agostinho, A. A. 1999. Determination of patterns of ichthyofauna co-occurence in the Paraná river basin, area of influence of the Itaipu reservoir. Interciencia 24(6):360-365

Benedito-Cecílio, E.; Agostinho, A. A.; Júlio Jr., H. F. \& Pavanelli, C. S. 1997. Colonização ictiofaunística do reservatório de Itaipu e áreas adjacentes. Revista Brasileira de Zoologia 14(1): $1-14$

Benine, R. C. 2004. Fish Base. Disponível em: <www. fishbase.org/ Moenkhausia_dichroura>. Acesso em: 12.12.2008.

Benine, C. R.; Castro, R. M. \& SABino, J. 2004. Moenkhausia bonita: A new small Characin fish from the rio Paraguay basin, southwestern Brazil (Characiformes: Characidae). Copeia 2004(1):68-73

Cassemiro, F. A.; Hahn, N. S. \& Rangel, T. F. L. V. 2003. Diet and trophic ecomorphology of the silverside, Odontesthes bonariensis, of the Salto Caxias reservoir, rio Iguaçu, Paraná, Brazil. Neotropical Icththyology 1(2):127-132.

Ferreira, E. J. G. 1984. Ictiofauna da represa hidrelétrica de Curuá-Una, Santarém, Pará. II - Alimentação e hábitos alimentares das principais espécies. Amazoniana 9(1):1-16.

Gerking, S. D. 1994. Feeding Ecology of Fish. San Diego, Academic. $416 \mathrm{p}$.

HAhN, N. S. \& Fugi, R. 2007. Alimentação de peixes em reservatórios brasileiros: alterações e consequiências nos estágios iniciais do represamento. Oecologia Brasiliensis 11(4): $469-480$

Hellawel, J. M. \& Abel, R. A. 1971. A rapid volumetric method for the analysis of the food of fishes. Journal of Fish Biology 3(1):29-37.
Hill, M. O. \& Gauch JR., H. G. 1980. Detrended correspondence analysis, an improved ordination technique. Vegetatio 42:47-58.

Hyslop, E. J. 1980. Stomach contents analysis, a review of methods and their application. Journal of Fish Biology 17:411-429

Júlio JR., H. F.; Bonecker, C. C. \& Agostinho, A. A. 1997. Reservatório de Segredo e sua inserção na bacia do rio Iguaçu. In: Agostinho, A. A. \& Gomes, L. C. eds. Reservatório de Segredo: bases ecológicas para o manejo. Maringá, EDUEM. p.1-17.

Lansac-TôHa, F. A.; Lima, A. F.; Hahn, N. S. \& Andrian, I. F. 1991. Composição da dieta alimentar de Hypophthalmus edentatus Spix, 1892 (Pisces, Hypophthalmidae) no reservatório de Itaipu e no rio Ocoí. Revista Unimar 13(2):147-162.

MacCune, B.; Mefford, M. J. 1999. PC-ORD: multivariate analysis of ecological data. Version 4.01. Oregon, $\mathrm{MjM}$ Software, Gleneden Beach.

Motta, P. J.; Clifton, K. B.; Hernandez, P. \& Eggold, B. T. 1995 Ecomorphological correlates in ten species of subtropical seagrass fishes: diets and microhabitat utilization. Environmental Biology of Fishes 44:37-60.

PAULY, D. 1980. On the interrelationships between natural mortality, growth parameters and mean environmental temperature in 175 fish stocks. Journal du Conseil International Pour I'Exploration de la Mer 39(3):175-92.

Peretti, D. \& Andrian, I. F. 2004. Trophic structure of fish assemblages in five permanent lagoons of the high Paraná River floodplain, Brazil. Environmental Biology of Fishes 71(1):95-103.

Poullly, M; Barrera, S. \& Rosales, C. 2006. Changes of taxonomic and trophic structure of fish assemblages along an environmental gradient in the upper Beni watershed (Bolívia). Journal of Fish Biology 68(1):137-156

Poullly, M.; Lino, F.; Bretenoux, J. G. \& Rosales, C. 2003. Dietarymorphological relationships in a fish assemblage of the Bolivian Amazonian floodplain. Journal of Fish Biology 62(5):1137-1158

Poullly, M.; Yonoki, T.; Rosales, C. \& Torres, L. 2004. Trophic structure of fish assemblages from Mamoré River floodplain lakes (Bolivia). Ecology of Freshwater Fish 13(4):245 257.

Rejas, D.; Villarpando, P. \& Carvajal, F. 2005. Variaciones estacionales en la dieta de Moenkhausia dichroura Kner (Pisces, Characidae) en una laguna de la várzea Del rio Ichilo (Cochabamba-Bolivia). Revista Boliviana de Ecologia y Conservación Ambiental 17:49-54.

Rocha, O.; Matsumura-Tundisi, T.; Espíndola, E. L. G.; Roche, K. F \& RietzleR, A. C. 1999. Ecological theory applied to reservoir zooplankton. In: Tundisi, J. G. \& Straskraba, M. S. eds. Theoretical Reservoir Ecology and its application. São Carlos, IIE - International Institute of Ecology. p.457-476.

Rocha, O.; Sendacz, S. \& Matsumura-Tundisi, T. 1995. Composition, biomass and productivity of zooplankton in natural lakes and reservoirs of Brazil. In: Tundisi, J. B.; Bicudo, C. E. \& Matsumura-Tudisi, T. eds. Limnology in Brazil. Rio de Janeiro, ABC/SLB. p.151-165.

Santos, G. M. 1995. Impactos da hidrelétrica Samuel sobre as comunidades de peixes do rio Jamari (Rondônia, Brasil). Acta Amazônica 25(3-4):247-280

SAzIMA, I. 1986. Similarities in feeding behaviour between some marine and freshwater fishes in two tropical communities. Journal of Fish Biology 29(1):53-65.

Woynarovich, E. 1991. The Hydroelectric power plants and the fish fauna. Verhandlungen Der Internationalen Vereinigung für Theoretische und Angewandte Limnologie 24:2531-2536. 